\title{
Biologia populacional de Pachyurus bonariensis Steindachner, 1879 (Perciformes, Sciaenidae), uma espécie alóctone no sistema hidrográfico da laguna dos patos, Brasil
}

\author{
Ana Paula Sassanovicz Dufech ${ }^{1,2}$ \& Clarice Bernhardt Fialho ${ }^{1}$ \\ Biota Neotropica v7 (n1) - http://www.biotaneotropica.org.br/v7n1/pt/abstract?article+bn03207012007 \\ Recebido em 04/10/05 \\ Versão Reformulada Recebida em 28/08/06 \\ Publicado em 29/03/06

\begin{abstract}
${ }^{1}$ Departamento de Zoologia, Laboratório de Ictiologia, Universidade Federal do Rio Grande do Sul, ${ }^{2}$ Corresponding author: Ana Paula Sassanovicz Dufech,
\end{abstract} \\ Av. Bento Gonçalves, 9500, CEP 90540-000, Porto Alegre, RS, Brazil, e-mail: cbfialho@ pro.via-rs.com.br \\ e-mail: apdufech@hotmail.com,www.ufrgs.br/ictio
}

\begin{abstract}
Dufech, A.P.S. \& Fialho, C.B. Population biology of Pachyurus bonariensis Steindachner, 1879 (Perciformes, Sciaenidae), an allochthonous species in the Laguna dos Patos hydrographic system, Brazil. Biota Neotrop. Jan/Apr 2007 vol. 7, no. 1 http://www.biotaneotropica.org.br/v7n1/pt/abstract?article+bn03207012007 ISSN 1676-0603.

The genus Pachyurus is widely distributed in the large hydrographic systems of South America, being represented only by Pachyurus bonariensis in the southern portion of the continent, in the La Plata drainage (Uruguay, Parana and Paraguay rivers). The occurrence of this species is recent in the Laguna dos Patos system, and most aspects about population biology and distribution of $P$. bonariensis for this region are unknown. Abundance, constancy of occurrence, sexual dimorphism and sex ratio were analyzed for the species. The establishment of this species in the Laguna dos Patos system is discussed. One hundred and one specimens ( 82 females, 15 males and four immature) were captured in the Parque Estadual de Itapuã, Rio Grande do Sul State, at Praia das Pombas, Lago Guaíba. The species was constant during the studied period, from June 2002 to July 2003. The abundance was higher in January and April 2003. Females were larger and significantly more abundant than males $\left(\chi^{2}=43.12 ; \mathrm{p}<0.05\right)$. These results and the identification of sexually mature females suggest reproductive activity and indicate that $P$. bonariensis is an allochthonous species that established a successful population in the Laguna do Patos hydrographic system.

Keywords: fish, Maria-Luiza, southern Brazil, introduction.

Resumo

Dufech, A.P.S. \& Fialho, C.B. Biologia populacional de Pachyurus bonariensis Steindachner, 1879 (Perciformes, Sciaenidae), uma espécie alóctone no sistema hidrográfico da Laguna dos Patos, Brasil. Biota Neotrop. Jan/Apr 2007 vol. 7, no. 1 http://www.biotaneotropica.org.br/v7n1/pt/abstract?article+bn03207012007 ISSN 1676-0603.

O gênero Pachyurus é amplamente distribuído nas maiores bacias hidrográficas da América do Sul, sendo representado na região sul do continente somente pela espécie Pachyurus bonariensis, na bacia do Prata (rios Uruguai, Paraná e Paraguai). A ocorrência desta espécie no sistema hidrográfico da laguna dos Patos é recente. Além disso, pouco se sabe acerca da biologia populacional e distribuição de $P$. bonariensis nessa região. Nesse sentido, foram analisados os seguintes aspectos populacionais da espécie: abundância, constância de ocorrência, dimorfismo sexual e proporção sexual. Discute-se ainda, o estabelecimento de P. bonariensis no sistema hidrográfico da laguna dos Patos. Os espécimes foram amostrados na praia das Pombas, lago Guaíba, Parque Estadual de Itapuã, Estado do Rio Grande do Sul. Foram capturados 101 indivíduos, sendo 82 fêmeas, 15 machos e quatro sexualmente imaturos. A espécie foi constante ao longo do período de estudo (junho de 2002 a julho de 2003), sendo sua abundância mais elevada em janeiro e abril de 2003. As fêmeas foram maiores e significativamente mais abundantes que os machos $\left(\chi^{2}=43,12 ; \mathrm{p}<0,05\right)$. Esses resultados, juntamente com a identificação de fêmeas sexualmente maduras, sugerem atividade reprodutiva desta espécie, indicando que $P$. bonariensis é uma espécie alóctone que se estabeleceu com sucesso no sistema da laguna dos Patos.
\end{abstract}

Palavras-chave: peixes, Maria-Luiza, sul do Brasil, introdução. 


\section{Introdução}

Na América do Sul, os gêneros Pachypops, Pachyurus, Petilipinnis e Plagioscion, pertencentes à família Sciaenidae, são endêmicos às águas continentais, podendo ocorrer eventualmente em estuários (Casatti 2003). Dentre eles, Pachyurus é o que apresenta maior número de espécies nominais (11 registradas até o momento) e maior área de ocorrência geográfica, estando amplamente distribuído nos sistemas dos rios Orinoco, Amazonas, Paraná-Paraguai-Uruguai, São Francisco, rios costeiros do leste do Brasil e rios das Guianas. Na região Amazônica, algumas de suas espécies são de grande importância na pesca artesanal de subsistência (Casatti 2001).

Conhecidas popularmente como Maria-luiza, as espécies do gênero Pachyurus são amplamente distribuídas em sistemas hidrográficos brasileiros, estando representado no Rio Grande do Sul (RS) pela espécie $P$. bonariensis Steindachner 1879. Esta espécie distribui-se pelos rios do sistema Paraná-Paraguai-Uruguai, à jusante do reservatório de Itaipu, incluindo Brasil, Paraguai, Uruguai e Argentina (Casatti 2001). A primeira ocorrência no sistema da laguna dos Patos, RS, foi registrada por Pinto et al. (2001), sendo interpretada como resultado da construção de canais de irrigação para cultivos de arroz ou em decorrência de sua introdução por pescadores e/ou piscicultores.

A ocorrência desta espécie no sistema da laguna dos Patos a caracteriza como alóctone, ou seja, uma espécie nativa de bacia hidrográfica brasileira registrada em bacia onde não ocorria naturalmente (IBAMA, Portaria 145/1998). Outros exemplos de espécies de peixes alóctones à laguna dos Patos são Trachelyopterus lucenai Bertoleti, da Silva \& Pereira 1995, amplamente disseminada neste sistema, e Acestrorhynchus pantaneiro Menezes 1992, registrado recentemente na região do Delta do Jacuí (Saccol-Pereira et al. 2006). Ambas as espécies também eram registradas no Rio Grande do Sul somente para a bacia do rio Uruguai.

Pachyurus bonariensis (Figura 1) é uma espécie demersal de pequeno porte entre as dominantes na maioria dos ambientes aquáticos tropicais, principalmente aqueles submetidos a impacto, como os represamentos estudados por Benedito-Cecílio \& Agostinho (1997). Agostinho \& Júlio (1999) citam a distribuição desta espécie como moderada, sendo encontrada somente à jusante do reservatório de Itaipu.

Esta espécie associa-se a ambientes lênticos sendo citada para a ictiofauna do Médio Paraná (Bonetto et al. 1965), e seu hábito alimentar é basicamente herbívoro (Panattieri \& Del Barco 1981) ou onívoro, com a dieta composta por insetos, crustáceos, oligoquetas e ovos (Lopez \& Castello 1997).

Este trabalho teve como objetivos analisar aspectos populacionais de $P$. bonariensis, tais como abundância, constância de ocorrência, dimorfismo sexual e proporção sexual. Com base nos resultados obtidos e em dados compilados por meio da análise de estudos recentes realizados nesta região, foi realizada uma discussão sobre o

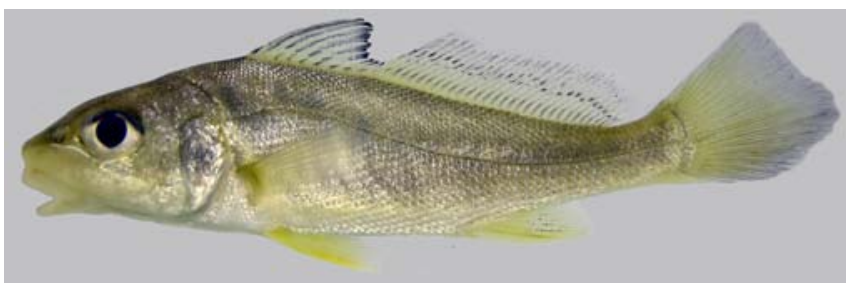

Figura 1. Exemplar de Pachyurus bonariensis (UFRGS 5712; $89 \mathrm{~mm} \mathrm{CP).}$ Foto: Luiz R. Malabarba.

Figure 1. Specimen of Pachyurus bonariensis (UFRGS 5712; $89 \mathrm{~mm} \mathrm{SL).}$ Photo: Luiz R. Malabarba. estabelecimento desta espécie alóctone no sistema hidrográfico da laguna dos Patos, no Estado do Rio Grande do Sul.

\section{Material e Métodos}

\section{1. Área de estudo}

O presente estudo foi desenvolvido no Parque Estadual de Itapuã, município de Viamão, Rio Grande do Sul. O Parque Estadual de Itapuã é uma reserva ecológica criada em 1973, apresentando a última amostra do ecossistema e paisagem original da região metropolitana de Porto Alegre, com campos, dunas, lagos, lagoas, ilhas, praias e morros às margens do lago Guaíba e da laguna dos Patos. Os sistemas hídricos do Parque inserem-se no sistema hidrográfico da laguna dos Patos, estando o Pontal de Itapuã localizado na margem esquerda da conjunção do lago Guaíba com a laguna dos Patos. A região de Itapuã é limnologicamente de águas doces, no entanto, ocorre certa influência de águas de origem marinha, provenientes do canal de ligação da laguna dos Patos, ao sul, com o mar em Rio Grande (Volkmer-Ribeiro 1981).

O local de coleta está situado nas imediações do trapiche da

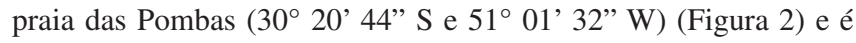
caracterizado por apresentar águas livres e claras, fundo arenoso e profundidade média de 1,5 m. A vegetação das margens é a mata de restinga, representada principalmente por sarandis (Cephalanthus glabratus), corticeiras-do-banhado (Erythrina cristagalli), maricás (Mimosa bimucronata) e juncos (Cyperus spp. e Scirpus spp.). A praia das Pombas apresenta forma de baía, sendo banhada pelas águas do lago Guaíba, que compreende um dos mais importantes recursos hidrográficos do Estado. O lago Guaíba é formado pelos rios Jacuí, Sinos, Caí e Gravataí e apresenta uma profundidade média de dois metros, estendo-se desde o Delta do Jacuí, ao Norte, até o Pontal de Itapuã (no Farol), ao Sul, onde apresenta profundidade máxima de $31 \mathrm{~m}$ (Menegat et al. 1998).

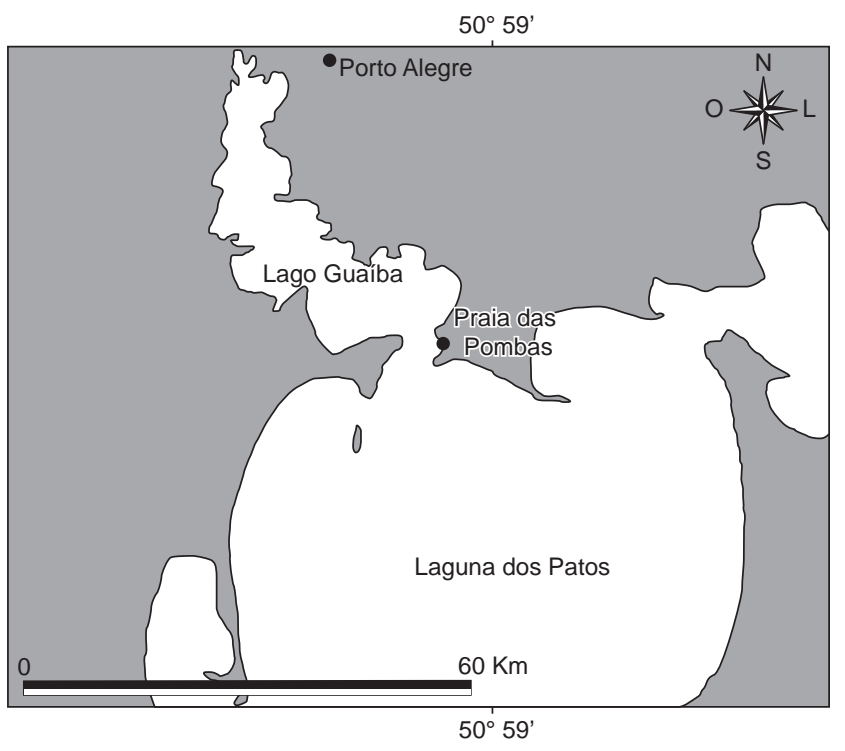

Figura 2. Mapa da área de estudo indicando o ponto de coleta na praia das Pombas, Parque Estadual de Itapuã, Rio Grande do Sul, Brasil.

Figure 2. Map of the study area showing the sample station at Praia das Pombas, Parque Estadual de Itapuã, Rio Grande do Sul State, Brazil. 


\section{Amostragens}

As amostragens foram realizadas mensalmente, entre $12 \mathrm{e}$ 17 horas, de junho de 2002 a julho de 2003, com exceção do mês de dezembro. Os indivíduos foram capturados com rede de arrasto do tipo picaré (10 $\mathrm{m}$ de comprimento, $2,5 \mathrm{~m}$ de altura, $5 \mathrm{~mm}$ entrenós adjacentes), com um esforço de captura de cinco arrastos por amostragem, e uma bateria de redes de espera (20 m de comprimento; $1,5 \mathrm{~m}$ de altura; 15, 20, 25, 30, $35 \mathrm{~mm}$ entrenós adjacentes), as quais eram dispostas a uma distância de aproximadamente dois metros da margem, onde permaneciam durante cinco horas.

Os indivíduos capturados foram fixados em formalina $10 \%$ e em laboratório, transferidos para etanol $70^{\circ} \mathrm{GL}$. De cada indivíduo foram registrados dados de comprimento padrão (CP, em milímetros), peso total (Wt, em gramas) e sexo, identificado por meio da análise macroscópica das gônadas. Os espécimes testemunhos deste estudo estão depositados na Coleção de Peixes do Departamento de Zoologia da Universidade Federal do Rio Grande do Sul (UFRGS 5712).

\section{Análise dos dados}

A abundância da espécie foi representada pelo número de indivíduos. Para o estudo da variação temporal esses valores, juntamente com os dados de biomassa, foram analisados mensalmente. A Constância de Ocorrência (Dajoz 1978) foi calculada com uso da fórmula: $\mathrm{C}=(\mathrm{P} / \mathrm{Q}) \times 100$ onde, $\mathrm{C}=$ constância de ocorrência da espécie; $\mathrm{P}=$ número de amostras em que a espécie ocorreu e $\mathrm{Q}=$ número total de amostras, podendo ser classificada em constante $(>50 \%)$, acessória $(25 \geq 50 \%)$ ou acidental $(\leq 25 \%)$.

Para a análise da estrutura em tamanho foram obtidas as distribuições das frequiências relativas (\%) das classes de comprimento de machos e fêmeas. A amplitude das classes de comprimento foi estabelecida através da regra de Sturges (Vieira 1991). Às distribuições de freqüências de classes de comprimento padrão de machos e fêmeas, foi aplicado o teste não paramétrico de Kolmogorov-Smirnov (Siegel 1975) para verificar a ocorrência ou não de dimorfismo sexual em relação ao tamanho atingido por ambos os sexos. A proporção sexual foi estimada através das freqüências de machos e fêmeas. Os resultados foram testados através do $\chi^{2}$ com o emprego, quando necessário, da correção de Yates (Zar 1999) para a verificação da existência ou não de diferenças significativas na proporção entre os sexos.

Os mapas foram plotados com o auxílio do software ArcView GIS versão 3.1.

\section{Resultados e Discussão}

\section{Aspectos populacionais}

Foram capturados 101 indivíduos de $P$. bonariensis (82 fêmeas, 15 machos e quatro indivíduos imaturos), sendo 19 espécimes com picaré e 82 com redes de espera. A espécie foi constante ao longo do período de estudo, ocorrendo em $92,3 \%$ dos meses estudados, podendo $P$. bonariensis ser considerada como uma espécie residente na praia das Pombas. Matthews (1998) afirma que as espécies constantes em todas as coletas fazem parte da comunidade aquática ali presente, sendo assim, podemos considerar que $P$. bonariensis foi componente real da assembléia de peixes da praia das Pombas. Majolo (2005), estudando a comunidade de peixes no rio Taquari (RS) relata que esta espécie foi constante, sendo capturada em aproximadamente $70 \%$ das amostras.

A abundância da espécie ao longo dos meses foi mais elevada em janeiro e abril de 2003 (Figura 3). A biomassa não apresentou um padrão bem definido, com maiores valores alcançados em junho e outubro de 2002 e janeiro, abril e maio de 2003 (Figura 4). A abun-

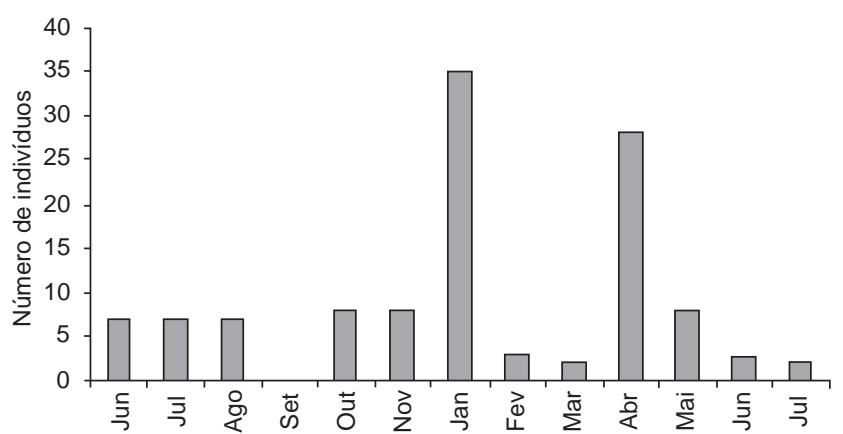

Figura 3. Variação mensal na abundância de Pachyurus bonariensis coletados na praia das Pombas, entre junho de 2002 e julho de 2003.

Figure 3. Monthly variation in abundance of Pachyurus bonariensis collected from Praia das Pombas, from June 2002 to July 2003.

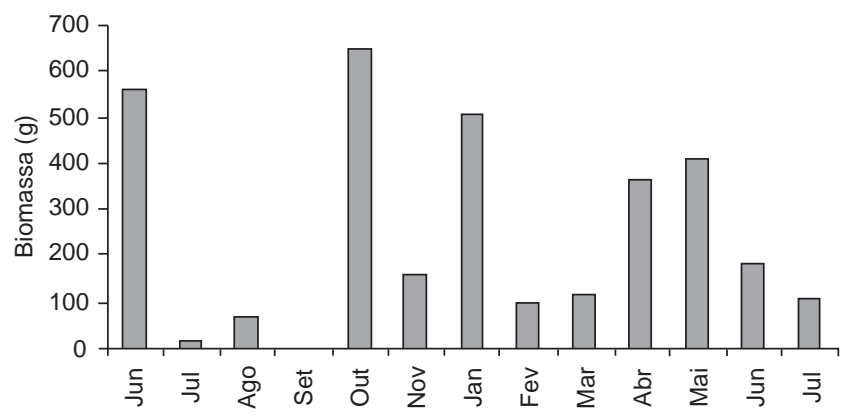

Figura 4. Variação mensal da biomassa (g) dos indivíduos de Pachyurus bonariensis, capturados na praia das Pombas entre junho de 2002 e julho 2003

Figure 4. Monthly variation in biomass ( $\mathrm{g}$ ) of specimens of Pachyurus bonariensis collected from Praia das Pombas, from June 2002 to July 2003.

dância da espécie foi também relatada por Braun (2005) e Milani (2005) em outra região deste sistema lagunar, sendo que no período 2003/2004, esta espécie contribuiu para uma importante parcela do estoque pesqueiro com valor comercial da região de Palmares do Sul, no Estado do Rio Grande do Sul.

A abundância de peixes em muitos ambientes de clima temperado mostra valores máximos durante os meses quentes (Allen \& Horn 1975, Day et al. 1981, Moyle et al. 1986). No presente estudo, as elevadas abundâncias de $P$. bonariensis nesse período coincidem com o período reprodutivo citado para a espécie, que ocorre de outubro/novembro e março (Braun 2005). Ciclos temporais na composição, abundância e distribuição das espécies de peixes podem ser explicados como uma estratégia dos peixes em explorar os recursos do meio, com o intuito de garantir o sucesso reprodutivo, uma vez que a maioria das espécies de peixes se reproduz na primavera e no verão (Fialho 1998).

A Figura 5 apresenta os registros de Pachyurus bonariensis no sistema da laguna dos Patos. Os locais de coleta, juntamente com as coordenadas geográficas, estão listados na Tabela 1. A espécie encontra-se amplamente distribuída ao longo do Sistema, sendo registrada mais ao sul na localidade de Arambaré e ao norte, em Arroio do Meio (rio Taquari). Agostinho et al. (2003) estudando a ictiofauna do rio as Antas, situado a montante do rio Taquari, não amostraram nenhum indivíduo desta espécie, o que sugere que $P$. bonariensis está em processo progressivo de conquista de ambientes no sentido sul-norte deste sistema hidrográfico. 
Tabela 1. Registros de Pachyurus bonariensis no sistema hidrográfico da laguna dos Patos, Rio Grande do Sul. Coleções: MCP = Museu de Ciências e Tecnologia da Pontifícia Universidade Católica do Rio Grande do Sul; MNRJ = Museu Nacional do Rio de Janeiro; UFRGS = Universidade Federal do Rio Grande do Sul.

Table 1. Records of Pachyurus bonariensis in the Laguna dos Patos hydrographic system, Rio Grande do Sul State. Collections: MCP = Museu de Ciências e Tecnologia da Pontifícia Universidade Católica do Rio Grande do Sul; MNRJ = Museu Nacional do Rio de Janeiro; UFRGS = Universidade Federal do Rio Grande do Sul.

\begin{tabular}{|c|c|c|c|c|}
\hline Registro & Ano & Localidade & Latitude / Longitude & Fonte \\
\hline 1 & 2000 & Barra do Ribeiro - lago Guaíba & $30^{\circ} 17^{\prime} 00^{\prime \prime} / 51^{\circ} 18^{\prime} 00^{\prime \prime}$ & UFRGS 4951 \\
\hline 2 & 2000 & Montenegro - rio Caí & $29^{\circ} 42^{\prime} 00^{\prime \prime} / 51^{\circ} 28^{\prime} 00^{\prime \prime}$ & MCP 26520 \\
\hline 3 & 2001 & Farol de Itapuã - lago Guaíba & $30^{\circ} 22^{\prime} 34^{\prime \prime} / 51^{\circ} 04^{\prime} 53^{\prime \prime}$ & MCP 27437 \\
\hline 4 & 2001 & Saco da Pólvora - Delta do Jacuí & $30^{\circ} 01^{\prime} 26^{\prime \prime} / 51^{\circ} 14^{\prime} 37^{\prime \prime}$ & MCP 27443 \\
\hline 5 & 2001 & Morro da Ponta Grossa - lago Guaíba & $30^{\circ} 10^{\prime} 43^{\prime \prime} / 51^{\circ} 14^{\prime} 04^{\prime \prime}$ & MCP 28051 \\
\hline 6 & 2001 & Taquara - rio dos Sinos & $29^{\circ} 41^{\prime} 11^{\prime \prime} / 50^{\circ} 51^{\prime} 03^{\prime \prime}$ & MCP 28076 \\
\hline 7 & 2002 & Praia das Pombas - lago Guaíba & $30^{\circ} 20^{\prime} 44^{\prime \prime} / 51^{\circ} 01^{\prime} 32,6^{\prime \prime}$ & UFRGS 5446 \\
\hline 8 & 2003 & Arambaré - laguna dos Patos & $30^{\circ} 57^{\prime} 07^{\prime \prime} / 51^{\circ} 30^{\prime} 01^{\prime \prime}$ & UFRGS 5884 \\
\hline 9 & 2003 & Foz do arroio Celupa - lago Guaíba & $30^{\circ} 06^{\prime} 10^{\prime \prime} / 51^{\circ} 18^{\prime} 42,4^{\prime \prime}$ & UFRGS 6373 \\
\hline 10 & 2003 & Lagoa do Casamento - laguna dos Patos & $30^{\circ} 26^{\prime} 22,83^{\prime \prime} / 50^{\circ} 38^{\prime} 38,36^{\prime \prime}$ & Braun (2005) \\
\hline 11 & 2003 & Lagoa dos Gateados - laguna dos Patos & $30^{\circ} 28^{\prime} 46,55^{\prime \prime} / 50^{\circ} 39^{\prime} 05,25^{\prime \prime}$ & Braun (2005) \\
\hline 12 & 2003 & Ponta dos Quatis - lago Guaíba & $30^{\circ} 14^{\prime} 56^{\prime \prime} / 51^{\circ} 09^{\prime} 09^{\prime \prime}$ & MNRJ 25614 \\
\hline 13 & 2004 & Ilha do Andrade - rio Taquari & $29^{\circ} 24^{\prime} 17,28^{\prime \prime} / 51^{\circ} 56^{\prime} 10,14^{\prime \prime}$ & Majolo (2005) \\
\hline 14 & 2005 & Saco do Ferraz - Delta do Jacuí & $30^{\circ} 00^{\prime} 34^{\prime \prime} / 51^{\circ} 14^{\prime} 41,3^{\prime \prime}$ & UFRGS 7584 \\
\hline 15 & 2006 & Praia de Fora - laguna dos Patos & $30^{\circ} 23^{\prime} 14,4^{\prime \prime} / 51^{\circ} 01^{\prime} 21^{\prime \prime}$ & UFRGS 8275 \\
\hline 16 & $2005-2006$ & Foz do arroio Dilúvio - lago Guaíba & $30^{\circ} 02^{\prime} 37,2^{\prime \prime} / 51^{\circ} 14^{\prime} 09,4^{\prime \prime}$ & A.P.S. Dufech (dados não publicados) \\
\hline 17 & 2005-2006 & Ilha da Pólvora - lago Guaíba & $30^{\circ} 01^{\prime} 19,1^{\prime \prime} / 51^{\circ} 14^{\prime} 34,7^{\prime \prime}$ & A.P.S. Dufech (dados não publicados) \\
\hline 18 & 2005-2006 & Ilha do Lino - rio Jacuí & $29^{\circ} 56^{\prime} 34^{\prime \prime} / 51^{\circ} 16^{\prime} 46^{\prime \prime}$ & A.P.S. Dufech (dados não publicados) \\
\hline 19 & 2005-2006 & Praia de Paquetá - rio dos Sinos & $29^{\circ} 56^{\prime} 02,1^{\prime \prime} / 51^{\circ} 14^{\prime} 05,7^{\prime \prime}$ & A.P.S. Dufech (dados não publicados) \\
\hline 20 & $2005-2006$ & Eldorado do Sul - lago Guaíba & $30^{\circ} 00^{\prime} 53,73^{\prime \prime} / 51^{\circ} 18^{\prime} 09,81^{\prime \prime}$ & A.P.S. Dufech (dados não publicados) \\
\hline 21 & 2005-2006 & Saco Santa Cruz - lago Guaíba & $30^{\circ} 01^{\prime} 18,2^{\prime \prime} / 51^{\circ} 16^{\prime} 50,9^{\prime \prime}$ & A.P.S. Dufech (dados não publicados) \\
\hline
\end{tabular}

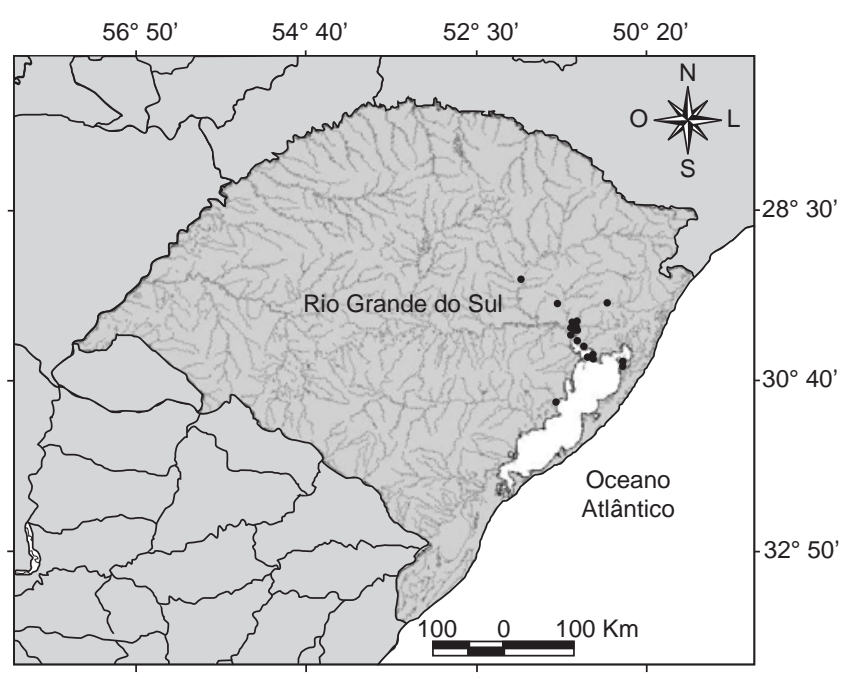

Figura 5. Registros de Pachyurus bonariensis no sistema hidrográfico da laguna dos Patos, Rio Grande do Sul, Brasil.

Figure 5. Records of Pachyurus bonariensis in the Laguna dos Patos hydrographic system, Rio Grande do Sul State, Brazil.

O comprimento padrão das fêmeas de $P$. bonariensis capturados na praia das Pombas variou de $74,1 \mathrm{~mm}$ a $205 \mathrm{~mm}$ e dos machos de $38,66 \mathrm{~mm}$ a $165 \mathrm{~mm}$. Foram estabelecidas sete classes de comprimento padrão, com um intervalo de $26,05 \mathrm{~mm}$. A classe com maior frequiência de indivíduos foi entre 64,71 e 90,75 mm (Figura 6). As

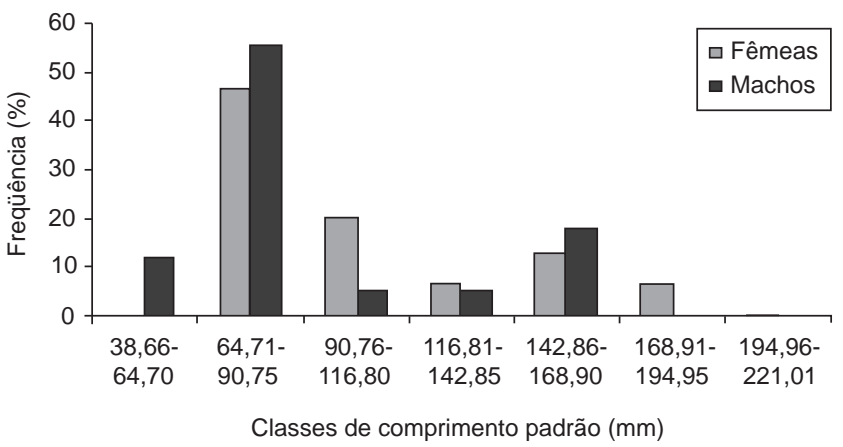

Figura 6. Frequiência relativa das classes de comprimento padrão para machos e fêmeas de Pachyurus bonariensis capturados na praia das Pombas, entre junho de 2002 e julho de 2003.

Figure 6. Relative frequency of the standard length classes of males and females of Pachyurus bonariensis collected from Praia das Pombas, from June 2002 to July 2003.

fêmeas foram mais abundantes nas maiores classes de comprimento, no entanto, não houve diferenças significativas quanto ao tamanho entre os sexos $\left(\chi^{2}=2,14 ; p>0,05\right)$. Braun (2005), estudando a biologia reprodutiva das espécies dominantes na lagoa do Casamento relata que as fêmeas de $P$. bonariensis foram significativamente maiores que os machos (fêmeas com comprimentos totais entre 80 e 264 mm e machos com comprimentos acima de $210 \mathrm{~mm}$ ).

O dimorfismo sexual, em termos de comprimento, tem ocorrência mais generalizada entre os Characiformes e Siluriformes e é caracte- 
rizado principalmente pelo maior tamanho alcançado pelas fêmeas, possivelmente relacionado a uma estratégia ligada à reprodução, visto que a fecundidade aumenta com o comprimento dos indivíduos (Agostinho \& Júlio 1999).

As fêmeas foram mais abundantes que os machos $\left(\chi^{2}=43,12\right.$; $\mathrm{p}<0,05)$, com razão sexual de aproximadamente 5:1. A proporção sexual e a estrutura de tamanho são informações básicas para o potencial reprodutivo e para a estimativa do tamanho do estoque das populações de peixes. Apesar de registrada ocasionalmente em algumas populações de peixes, uma proporção sexual de 1:1 é raramente esperada (Vicentini \& Araújo 2003) e esse desvio pode ser atribuído a diversas causas, tais como influência da temperatura na determinação do sexo (Conover \& Kynard 1981), mortalidade seletiva por sexo como predação diferencial, comportamento sexual diferenciado, taxa de crescimento ou expectativa de vida.

A estrutura de tamanho e sexual é específica, podendo haver variações intra e interpopulacionais decorrentes não apenas de seletividade, inerentes às artes de pesca, como também das condições ambientais, principalmente alimentares, as quais interferem no recrutamento, crescimento e mortalidade (Nikolski 1969). Outro fator que pode influenciar a proporção sexual é a disponibilidade de alimento. Nikolski (1963) relatou que onde o alimento é abundante, as fêmeas predominam e uma situação inversa pode ser observada em regiões onde o alimento é limitado.

As diferenças nas classes de comprimento padrão observadas para $P$. bonariensis, bem como a identificação de fêmeas sexualmente maduras (observação pessoal), sugerem atividade reprodutiva dos indivíduos desta espécie. Esta hipótese pode ser confirmada com os resultados obtidos por Braun (2005), onde foram evidenciados alevinos, jovens e adultos da espécie, além de um pico reprodutivo nos meses onde a temperatura foi mais elevada. Estes resultados indicam que $P$. bonariensis encontrou condições favoráveis para reprodução no sistema da laguna dos Patos.

\section{Introdução de espécies}

A introdução de peixes em ambientes naturais é um tema pouco difundido e de suma importância no que tange à conservação da ictiofauna nativa (Welcomme 1988). A introdução de peixes de água doce, em particular, tem sido documentada, em sua maioria, para ambientes aquáticos degradados em muitas áreas do mundo (Moyle \& Nichols 1973, Arthington et al. 1990, Brown 2000). No Brasil, a taxa de introdução de espécies de peixes é ainda crescente, em contraste com outras regiões do mundo (Agostinho \& Júlio 1996, Magalhães et al. 2002).

As conseqüências ou impactos que espécies exóticas ou alóctones ao sistema hidrográfico podem causar ao ambiente são inúmeras. Dentre os efeitos diretos destacam-se a extinção de espécies nativas (Usher 1988, Rapoport 1977); mudança na estrutura original da comunidade nativa (Moyle 1976, Li \& Moyle 1981, Balon \& Bruton 1986); competição por alimento, abrigo, sítios de desova e criação (Fryer 1961); predação (Zaret \& Paine 1973); introdução de parasitas ou doenças (Courtenay \& Meffe 1989, Ross 1991, Crowl et al. 1992) e hibridização (Couternay \& Stauffer 1984, Welcomme 1988, Kennedy 1993). As espécies introduzidas podem também afetar indiretamente a fauna nativa através da alteração nas condições do hábitat e/ou nos processos dos ecossistemas pelo forrageamento entre outras atividades (Taylor et al. 1984, Arthington \& McKenzie 1997).

Segundo Agostinho \& Júlio (1999), a corvina Plagioscion squamosissimus, uma espécie alóctone à bacia do alto Paraná e amplamente disseminada, apresenta importante participação na pesca do reservatório de Itaipu, sendo uma das espécies bem-sucedidas e adaptadas, a exemplo de Pachyurus bonariensis no sistema da laguna dos Patos. A coexistência entre espécies nativas e introduzidas pode facilitar perturbações abióticas naturais (Meffe 1984, Brown \& Moyle 1997). O número de ocasiões de introduções é um fator bastante importante que influencia o sucesso no estabelecimento de peixes, aves e outros organismos (Ross 1991, Cassey 2001). Uma vez estabelecida, a espécie introduzida pode ser citada, junto com a alteração do hábitat, como um dos maiores fatores que contribui para a extinção de muitas espécies de peixes (Miller et al. 1989).

Apesar disso, os resultados obtidos neste trabalho não comprovam nenhum impacto concreto a respeito da presença de Pachyurus bonariensis na laguna dos Patos, possível somente através de estudos mais aprofundados. Pode-se concluir que esta espécie alóctone encontra-se muito bem distribuída e adaptada ao sistema da laguna dos Patos, pois foi registrada sua atividade reprodutiva, sendo também considerada como espécie de importância comercial em algumas regiões do Estado.

\section{Agradecimentos}

Ao Prof. Dr. Luiz Roberto Malabarba pelas sugestões no decorrer do trabalho e aos colegas do Laboratório de Ictiologia (UFRGS) pelo auxílio nas saídas de campo. Ao biólogo Maurício Tavares pelas sugestões e pela ajuda na elaboração dos mapas. Ao $\mathrm{CNPq}$ (proc. 464545/00-5 e 476821/2003-7) e à CAPES pela bolsa concedida.

\section{Referências Bibliográficas}

AGOSTINHO, A.A. \& JÚLIO, H.F. 1996. Ameaça ecológica: peixes de outras águas. Ciência Hoje 124(21):36-44.

AGOSTINHO, A.A. \& JÚliO, H.F. 1999. Peixes da bacia do alto rio Paraná. In Estudos ecológicos de comunidades de peixes tropicais (R.H. Lowe-McConnell). EDUSP, São Paulo, p.374-400.

AGOSTINHO, A.A., LATINI, J.D., LUZ, K.D.G. \& GOMES, L.C. 2003. A ictiofauna do rio das Antas, área de influência do Complexo Energético Rio das Antas. EDUEM, Maringá.

ALLEN, L.G. \& HORN, M.H. 1975. Abundance, diversity and seasonality of fishes in Colorado lagoon, Alamitos Bay, California. Estuar. Coast. Mar. Sci. 3:371-380.

ARTHINGTON, A.H. \& MCKENZIE, F. 1997. Review of impacts of displaced/introduced fauna associated with inland waters. State of the Environment Technical Paper Series (Inland Waters), Department of the Environment, Canberra, Australia.

ARTHINGTON, A.H., HAMLET, S. \& BLUHDORN, D.R. 1990. The role of habitat disturbance in the establishment of introduced warm-water fishes in Australia. In Introduced and translocated fishes and their ecological effects (D.A. Pollard, ed.). Bureau of Rural Resources Proceedings (8). Australian Government Publishing Service, Canberra, p.61-66.

BALON, E.K. \& BRUTON, M.N. 1986. Introduction of alien species or why scientic advice is not heeded. Env. Biol. Fish. 16:225-230.

BENEDITO-CECÍlLIO, E. \& AGOSTINHO, A.A. 1997. Estrutura das populações de peixes do reservatório do Segredo. In Reservatório do Segredo: bases ecológicas para o manejo (A.A. Agostinho \& L.C. Gomes, eds.). EDUEM, Maringá, p.113-119.

BONETTO, A.A., PIGNALBERI, C. \& CORDIVIOLA, E. 1965. Contribución al conocimiento de las poblaciones de peces de las lagunas isleñas en el Paraná medio. Anais In Congresso Latino Americano de Zoologia, São Paulo, 2:131-144.

BRAUN, A.S. 2005. Biologia reprodutiva e identificação do uso de lagoa marginal como sítio de reprodução para espécies dominantes da ictiofauna da lagoa do Casamento, sistema nordeste da laguna dos Patos, RS. Tese de Doutorado, Pontifícia Universidade Católica do Rio Grande do Sul, Porto Alegre.

BROWN, L.R. 2000. Fish communities and their associations with environmental variables, lower San Joaquin River Drainage, California. Env. Biol. Fish. 57:251-269. 
BROWN, L.R. \& MOYLE, P.B. 1997. Invading species in the Eel River, California: successes, failures, and relationships with resident species. Env. Biol. Fish. 49:271-291.

CASATTI, L. 2001. Taxonomia do gênero Pachyurus Agassiz, 1831 (Teleostei: Perciformes: Sciaenidae) e descrição de duas novas espécies. Comum. Mus. Ciênc.Tecnol. PUCRS, série Zoologia 14(2):99-207.

CASATTI, L. 2003. Sciaenidae (drums or croakers). In Checklist of the freshwater fishes of the South and Central America (R.E. Reis, S.O. Kullander \& C.L. Ferraris, eds.). EDIPUCRS, Porto Alegre, p.599-602.

CASSEY, P. 2001. Determining variation in the success of New Zealand land birds. Global Ecol. Biogeog. 10:161-172.

CONOVER, D.O. \& KYNARD, M.H. 1981. Environmental sex determination: interaction of temperature and genotype in a fish. Nature 326:496-498.

COURTENAY JR., W.R. \& STAUFFER JR., J.R. 1984. Distribution, biology and management of exotic fishes. The Johns Hopkins University Press, Baltimore.

COURTENAY, W.R. \& MEFFE, G.K. 1989. Small fishes in strange places: a review of introduced poeciliids. In ecology and evolution of livebearing fishes (Poeciliidae) (G.K Meffe \& F.F. Snelson, eds.). Prentice Hall, Englewood Cliffs, p.319-331.

CROWL, T.A.,TOWNSEND, C.R. \& MCINTOSH, A.R. 1992. The impact of introduced brown and rainbow trout on native fish: the case of Australasia. Rev. Fish Biol. Fisheries 2:217-241.

DAJOZ, R. 1978. Ecologia Geral. Editora Vozes, Petrópolis.

DAY, J.H., BLABER, S.J.M. \& WALLACE, J.H. 1981. Estuarine ecology with particular reference to southern Africa. In Estuarine fishes (J.H. Day, ed.) A. A. Balkema, Rotterdam, p.199-221.

FIALHO, C.B. 1998. Estudo da comunidade de peixes da lagoa das Custódias, Tramandaí, Rio Grande do Sul, Brasil. Tese de Doutorado, Universidade Federal de São Carlos, São Carlos.

FRYER, G. 1961. Observations on the biology of the cichlid fish Tilapia variabilis in the northern waters of Lake Victoria (East Africa). Rev. Zool. Bot. Afr. 64:1-33.

KENNEDY, C.R. 1993. Introduction, spread and colonization of new localities by fish helminthes and crustacean parasites in the British Isles: a perspective and appraisal. J. Fish Biol. 43:287-301.

LI, H.W. \& MOYLE, P.B. 1981. Ecological analysis of species introductions into aquatic systems. Trans. Amer. Fish. Soc. 110:772-782.

LOPEZ, R. \& CASTELLO, J. 1997. Corvinas del Río de la Plata (Pisces, Sciaenidae). Comun. Mus. Argent. Cienc. Natural. Bernard. Rivad. $1: 1-12$.

MAGALHÃES, A.L.B., AMARAL, I.B., RATTON, T.F. \& BRITO, M.F.G. 2002. Ornamental exotic fishes in the Glória reservoir and Boa Vista stream, Paraiba do Sul river basin, state of Minas Gerais, southeastern Brazil. Comun. Mus. Ciênc. Tecnol. PUCRS, série Zoologia 15(2):265-278.

MAJOLO, M.A. 2005. Estudo da taxocenose de peixes na Ilha do Andrade, bacia hidrográfica do rio Taquari, Arroio do Meio, Rio Grande do Sul - Brasil. Dissertação de Mestrado, Universidade Federal do Rio Grande do Sul, Porto Alegre.

MATTEWS, W.J. 1998. Patterns in freshwaters fish ecology. Chapman \& Hall, New York.

MEFFE, G.K. 1984. Effects of abiotic disturbance on coexistence of predator-prey fish species. Ecology 65:1525-1534.

MENEGAT, R., PORTO, M.L., CARRARO, C.C. \& FERNANDES, L.A.D. 1998. Atlas Ambiental de Porto Alegre. EDUFRGS, Porto Alegre.

MILANI, P.C.C. 2005. Diagnóstico da pesca artesanal na lagoa do Casamento, sistema nordeste da laguna dos Patos: uma proposta de manejo. Dissertação de Mestrado, Pontifícia Universidade Católica do Rio Grande do Sul, Porto Alegre.

MILLER, R.R., WILLIAMS, J.D. \& WILLIAMS, J.E. 1989. Extinctions of North American fishes during the past century. Fisheries 14:22-38.

MOYLE, P.B. 1976. Fish introductions in California: history and impact on native fishes. Biol. Cons. 9:101-118.
MOYLE, P.B., DANIELS, R.A., HERBOLD, B.H. \& BALTZ, D.M. 1986. Patterns of distribution and abundance of a non-coevolved assemblage of estuarine fishes in California. Fish. Bull. 84:105-117.

MOYLE, P.B. \& NICHOLS, R.D. 1973. Ecology of some native and introduced fishes of the Sierra Nevada Foothills in Central California. Copeia 1973:478-490.

NIKOLSKI, G.V. 1963. The ecology of fishes. 6 ed., Academic Press, London.

NIKOLSKI, G.V. 1969. Theory of fish population dynamics. Oliver \& Boyd, Edimburg.

PANATTIERI, A. \& DEL BARCO, D. 1981. Peces de la Província de Santa Fé. Revista Cyta 20:30-33.

PINTO, R.F., OLIVEIRA, C.L.C., COLOMBO, P., FIALHO, C.B. \& MALABARBA, L.R. 2001. Primeiro registro de Pachyurus bonariensis (Steindachner, 1879) (Perciformes, Sciaenidae) para o sistema da Laguna dos Patos, Rio Grande do Sul, Brasil. Resumo In XIV Encontro Brasileiro de Ictiologia, Unisinos, São Leopoldo.

RAPOPORT. 1977. El desmanejo de las especies exóticas. San Carlos de Bariloche: Dep. de Recursos Naturales Y Energía, Fundación Bariloche.

ROSS, S.T. 1991. Mechanisms structuring stream fish assemblages: are there lessons from introduced species? Env. Biol. Fish. 30:359-368.

SACCOL-PEREIRA, A., MILANI, P.C.C. \& FIALHO, C.B. 2006. Primeiro registro de Acestrorhynchus pantaneiro Menezes 1992, (Characiformes, Acestrorhynchidae) no sistema da laguna dos Patos, Rio Grande do Sul, Brasil. Biota Neotropica v 6 (n3) - http://www.biotaneotropica.org. br/v6n3/pt/abstract?short-communication+bn00706032006

SIEGEL, S. 1975. Estatística não paramétrica. McGraw-Hill, São Paulo.

TAYLOR, J.N., COURTENAY, W.R. \& MCCANN, J.A. 1984. Known impact of exotic fishes in the continental United States. In Distribution, biology and management of exotic fishes (W.R. Courtenay \& J.R. Stauffer, eds.). Johns Hopkins University Press, Baltimore, p.322-373.

USHER, M.B. 1988. Biological invasions of nature reserves: a search for generalization. Biol. Conserv. 44:119-135.

VICENTINI, R.N. \& ARAÚJO, F.G. 2003. Sex ratio and size structure of Micropogonias furnieri (Desmarest, 1823) (Perciformes, Sciaenidae) in Sepetiba Bay, Rio de Janeiro, Brazil. Braz. J. Biol. 63(4):559-566.

VIEIRA, S. 1991. Introdução à Bioestatística. 2 ed., Editora Campus, Rio de Janeiro.

VOLKMER-RIBEIRO, C. 1981. Limnologia e a vegetação de macrófitas na lagoa Negra, Parque Estadual de Itapuã, Rio Grande do Sul. Iheringia, Série Botânica 27:38-59.

WELCOMME, R.L. 1988. Internacional introduction of inland aquatic species. FAO, Fisheries Technical Papers 294:1-318.

ZAR, J.H. 1999. Biostatistical Analysis. 4 ed. Northern Illinois University, Prentice-Hall.

ZARET, T.M. \& PAINE, R.T. 1973. Species introduction in a tropical lake. Science 182:445-449

Título: Biologia populacional de Pachyurus bonariensis Steindachner, 1879 (Perciformes, Sciaenidae), uma espécie alóctone no sistema hidrográfico da laguna dos patos, Brasil.

Author: Dufech, APS e Fialho, CB

Biota Neotropica, Vol.7 (número 1): 2007

http://www.biotaneotropica.org.br/v7n1/pt/abstract?article+ bn03207012007

Recebido em 04/10/05 - Versão reformulada recebida em 28/08/06 - Publicado em 29/03/06

ISSN 1676-0603 